\title{
Seismology of Solar Internal Rotation
}

\author{
Takashi Sekii \\ Solar Physics Division, National Astronomical Observatory of Japan, \\ Mitaka, Tokyo 181-8588, JAPAN
}

\begin{abstract}
Helioseismologists have been highly successful in revealing how the solar interior differentially rotates, through observations of rotationally split eigen $p$ modes of the Sun. What we currently know about the internal rotation of the Sun is reviewed, and outstanding issues are discussed.
\end{abstract}

\section{Introduction}

The 22-year solar activity cycle is believed to be caused by an interplay between magnetic field and internal flows, of which differential rotation seems to constitute the main component. The surface differential rotation of the Sun has been known for a long time, but observational study of the internal differential rotation was not possible until the era of helioseismology. Realistic numerical simulation is extremely difficult, owing to the large Reynolds number in the solar convection zone. Thus helioseismology provides an essential tool in probing the internal dynamics of our star. Seismological study of solar internal rotation is important not only in advancing our understanding of the solar cycle, but also in posing constraints on theory of the dynamical evolution of stars.

\section{Rotational splitting}

Rotation affects wave propagation in the Sun, thereby modifying frequencies of eigenmodes mainly via advection. The Sun is a slow rotator in the sense that the rotational period is much longer than the global dynamical time scale. Linear perturbation theory is therefore applicable. The frequency shift of a mode is then $m \times$ (average rotation rate), where $m$ is the azimuthal order of the mode. Observed shifts indicate that average rotational periods are of the order of a month, as is observed on the surface, justifying the linear treatment. The way the average is taken, expressed by a sensitivity function, differs from mode to mode. The sensitivity function, called splitting kernel, is calculable from an equilibrium model. Given a distribution of angular velocity, therefore, one can calculate the rotational shift of eigenfrequency for any eigenmode. This is sometimes called the forward problem. The inverse problem, then, is to find the underlying angular velocity distribution from an observed set of rotational shifts. 


\section{Inversion}

Since the forward problem is linear, the inverse problem can also be formulated as a linear problem. For discussion for linear inversion methodology see, e.g., Sekii (1997). Modern experiments, such as the GONG project and the SOHO satellite, have produced splitting data of unprecedented quality, which have subsequently been inverted to obtain angular velocity in the solar interior (Thompson et al. 1996; Schou et al. 1998). It was found that, throughout the convection zone, latitudinal differential rotation is similar to that of the surface. Near the base of the solar convection zone there is a shear layer, beneath which there is no strong differential rotation i.e. the rotation profile is consistent with that of a rigid rotation.

\section{Tachocline}

The position of the shear layer near the base of the convection zone, called tachocline (Spiegel and Zahn 1992), coincides with a sound speed anomaly found from sound speed inversions (Gough et al. 1996). This may be evidence for extra mixing caused by the shear (Gough \& McIntyre 1998), which circulates back the gravitationally settled helium to the convection zone, thereby reducing the local mean molecular weight. The dynamics of the tachocline is becoming one of the hottest topics in astrophysical fluid dynamics (e.g. Garaud 2003). Howe et al. (2000) examined temporal variation of the layer and reported a 1.3-year periodicity.

\section{Rotation in the convection zone}

In classical numerical simulations of the solar convection zone, as presented by Glatzmaier (1985), Taylor-Proudman type columnar rotation is produced in the low- to mid-latitudes. By ignoring the inertia term in the fluid equation of motion for a stationary flow, it can be shown that the $z$-gradient of rotational velocity is proportional to $\sin \chi$, where $z$ runs parallel to rotation axis and $\chi$ is the angle between density gradient and pressure gradient. For a barotropic fluid, $\chi$ vanishes and so does the $z$-gradient (Taylor-Proudman theorem). Helioseismic results show that this is not the case, and that the departure from the columnar rotation is in the sense of a warm pole. From the departure, we can estimate the angle $\chi$ and even compute the nominal latitudinal gradient of temperature $T$ along the constant pressure surface, by assuming the ideal gas equation of state. The result is that the relative temperature gradient $\partial \log T / \partial \phi$, where $\phi$ is colatitude, is of the order of $10^{-7}$ (Sekii 2003). The number is so small basically because the rotation is so slow. This departure must have been caused by terms ignored in the equation, such as the Reynolds stress. According to Elliott (2003), however, Reynolds stress alone cannot explain the departure. 


\section{Implication on dynamo}

Classical $\alpha-\omega$ dynamo models required a large radial gradient of angular velocity in the solar convection zone. According to the current view, however, there is no such gradient. This led to the idea of dynamo action in the tachocline region (Choudhuri 1990; Parker 1993). Dikpati \& Charbonneau (1999) were the first to carry out kinematic dynamo calculation by using the helioseismologically inferred rotation rate. Such models tend to produce butterfly diagrams with eruption too far from the equator. Nandy \& Choudhuri (2002) suggested that meridional flow penetrating into the radiative zone can solve the problem. This is of course so-called flux-transport dynamo.

Hathaway et al. (1996) found a surface pole-ward flow of up to $20 \mathrm{~m} / \mathrm{s}$ from GONG data. With time-distance technique applied to MDI data, Giles et al. (1997) found that such a flow persists down to the depth of $30 \mathrm{Mm}$ below the photosphere. In both cases, the flow was detected up to a latitude of around $60^{\circ}$. The biggest unanswered question concerns the properties of the counter flow, particularly in the context of a flux-transport dynamo. If the overall circulation satisfies anything like $|\rho v| \sim$ const. (cf. Gough \& Sekii 1998), then the counterflow velocity will be very small and difficult to detect.

\section{Polar Region}

Rotation inversions of MDI data indicated the presence of a jet, as well as an extra amount of slowing down towards the pole, in the high-latitude region (Schou et al. 1998). Global mode splitting is rather insensitive to this region, which renders it difficult to confirm these findings. Local-helioseismology may be the way, especially if the Sun can be viewed from a high-latitude. Such a proposition has indeed been made, for the Solar Orbitor mission (Gizon et al. 2001). The success of the local approach however has so far been confined to the near-surface layers and it remains to be seen how deep we can go with this new approach.

\section{Deep interior}

Without g-mode splitting data, we have to rely on low-degree p modes for investigation of deep core rotation. For low-degree modes, full disc, Sun-as-a-star observation seems to have an advantage over spatially resolved observation (and is more relevant to stellar case) but there is a problem of line blending: splitting measurement tends to be biased when line widths are comparable to rotational shifts. Also, there is an issue of poor localization due to lack of odd parity modes. Without these modes, the sensitivity function for combinations of $\ell=1$ modes, for example, have a different angular profile from the one from $\ell=2$ modes. Therefore, the rotation rate sampled by dipole modes and by quadrupole modes are difficult to disentangle. 


\section{Near Future}

Helioseismology has played a crucial role in revealing the dynamics of the solar interior. In the bulk of the solar convection zone we now know enough to tell that classical dynamo models do not work, which has inspired new models. Deep interior and polar region have been illusive and they may remain so for a while. Local helioseismology will give us further clues for the entire dynamical picture and this may not be confined to the surface layers alone.

Kawaler, Sekii \& Gough (1999) looked at data from the WET campaign on pulsating white dwarfs PG1159 (Winget et al. 1991) and GD358 (Winget et al. 1994), to find evidence for differential rotation. Recently, Aerts et al. (2003) reported evidence for non-rigid rotation in a $\beta$-Cephei variable HD129929. Application to stars has begun, which is challenging but doubtlessly worthwhile.

\section{References}

Aerts, C. et al. 2003, Science, 300, 1926

Choudhuri, A. R. 1990, ApJ, 355, 733

Dikpati, M., \& Charbonneau, P. 1999, ApJ, 518, 508

Elliott, J. R. 2003, in Stellar Astrophysical Fluid Dynamics, ed. M. J. Thompson \& J. Christensen-Dalsgaard (Cambridge University Press), 315

Garaud, P. 2003, in Stellar Astrophysical Fluid Dynamics, ed. M. J. Thompson \& J. Christensen-Dalsgaard (Cambridge, Cambridge University Press), 131

Giles, P. M. et al. 1997, Nature, 390, 52

Gizon, L., et al. 2001, in Solar Encounter: The First Solar Orbitor Workshop, ed. B. Battrick \& H. Sawaya-Lacoste (Noordwijk, ESA), 227

Glatzmaier, G. 1985, ApJ, 291, 300

Gough, D., \& McIntyre, M. E. 1998, Nature, 394, 755

Gough, D. O., \& Sekii, T. 1998, in Sounding Solar and Steller Interiors, ed. G. Berthomieu, J. Provost and F. X. Schmider (Nice, Observatoire de la Côte d'Azur), 93

Gough, D. O. et al. 1996, Science, 272, 1296

Hathaway, D. et al. 1996, Science, 272, 1306

Howe, R. et al. 2000, Science, 287, 2456

Kawaler, S. D., Sekii, T., \& Gough, D. O. 1999, ApJ, 516, 349

Nandy, D., \& Choudhuri, A. R. 2002, Science, 296, 1671

Parker, E. N. 1993, ApJ, 408, 707

Schou, J. et al. 1998, ApJ, 505, 390

Sekii, T. 1997, in Sounding Solar and Steller Interiors, ed. G. Berthomieu, J. Provost \& F. X. Schmider (Dordrecht, Kluwer Academic), 189

Sekii, T. 2003, in Stellar Astrophysical Fluid Dynamics, ed. M. J. Thompson \& J. Christensen-Dalsgaard (Cambridge, Cambridge University Press), 263

Spiegel, E. A., \& Zahn, J.-P. 1992, A\&A, 265, 106

Thompson, M. J. 1996, Science, 272, 1300

Winget, D. E. et al. 1991, ApJ, 378, 326

Winget, D. E. et al. 1994, ApJ, 430, 839 